\title{
Multiple Autoimmune Propensity and B-Non-Hodgkin Lymphoma: Cause or Effect?
}

\author{
E. Koumati, ${ }^{1}$ M. Palassopoulou, ${ }^{2}$ P. Matsouka, ${ }^{2}$ A. Polyzos, ${ }^{1}$ \\ G. N. Dalekos, ${ }^{1,3}$ and K. Zachou ${ }^{1,3}$ \\ ${ }^{1}$ Department of Medicine and Research Laboratory of Internal Medicine, School of Medicine, University of Thessaly, \\ Biopolis, Mezourlo, 41110 Larissa, Greece \\ ${ }^{2}$ Department of Haematology, School of Medicine, University of Thessaly, Biopolis, Mezourlo, 41110 Larissa, Greece \\ ${ }^{3}$ Institute of Biomedical Research and Technology, Centre for Research and Technology-Thessaly (CE.RE.TE.TH), Larissa, Greece
}

Correspondence should be addressed to G. N. Dalekos, dalekos@med.uth.gr

Received 7 January 2011; Accepted 1 March 2011

Academic Editor: Corrado Betterle

Copyright ( $\odot 2011$ E. Koumati et al. This is an open access article distributed under the Creative Commons Attribution License, which permits unrestricted use, distribution, and reproduction in any medium, provided the original work is properly cited.

\begin{abstract}
We report a case of multiple autoimmunity consisting of the presence of autoimmune haemolytic anaemia (AIHA), antimitochondrial antibodies (AMAs), and antiphospholipid antibodies (APLAbs) as the presenting manifestations of an extrahepatic B-non-Hodgkin lymphoma (B-NHL) in a 63-year-old woman. The patient presented with fatigue attributed to severe AIHA. Due to increased serum IgM and $\gamma$-GT levels, an investigation for AMA was performed, which proved positive with anti-M2 specificity. A prolongation of activated partial thromboplastin time (aPTT) led to the determination of APLAbs (lupus anticoagulant and other APLAbs) which were also positive. Bone marrow biopsy in combination with immmunohistochemical studies established the diagnosis of lymphoplasmacytic B-NHL. Ten months later, B-NHL was in remission while AMA and APLAbs were still positive. In conclusion, we documented the coexistence of multiple autoimmune reactions together with B-NHL highlighting the possible common pathogenetic pathways of the two entities.
\end{abstract}

\section{Introduction}

Lymphoproliferative disorders include several well-recognized disease entities, defined by different cell origin, pathol$\mathrm{ogy}$, and prognosis. Immune dysregulation is thought to play a major role in lymphomagenesis as attested by the increased risk of certain lymphomas following organ transplantation, infections, immunodeficiency states, and autoimmune diseases or syndromes [1-3].

On the other hand, autoimmune diseases comprise a broad variety of conditions characterized by dysregulation of the immune response leading to the loss of tolerance to self-antigens. Diverse associations between malignancy and autoimmunity have been shown in multiple levels $[2,4]$. In particular, the association between lymphomas and autoimmunity is well known several years ago, starting by observations that autoimmunity is often present in patients with non-Hodgkin lymphoma (NHL), mouse strains showing association between autoimmune disease and lymphoma, and epidemiologic evidence that autoimmune disorders carry a significantly increased risk of NHL development compared to healthy population $[1,5,6]$. Exploring the pathogenetic mechanisms of this association, recent data show that they might be bi-directional [7-9].

However, autoimmune rheumatic features and/or autoimmune phenomena occur frequently in the course of lymphoproliferative malignancies, and they may sometimes be the first sign of the malignancy [7]. However, to the best of our knowledge, multiple autoimmunity consisting of the presence of autoimmune haemolytic anaemia (AIHA), antimitochondrial antibodies (AMAs), and antiphospholipid antibodies (APLAbs) as the presenting manifestations of an extrahepatic B-NHL has not been previously described so far. Therefore, a representative case is reported herein, with an appropriate and extensive review of the literature.

\section{Case Report}

A 63-year-old woman was admitted to our department because of fatigue, weakness and dysesthesias (numbness) of 
the lower limbs during the last two months but without fever. Physical examination revealed paleness, mild jaundice, and splenomegaly but no palpable peripheral lymph nodes and no neurological findings. Her family and past history were unrevealing.

Laboratory tests revealed severe anaemia, increased reticulocyte count, and elevated serum levels of indirect bilirubin, gamma-glutamyl-transpeptidase $(\gamma$-GT), immunoglobulin M (IgM), and lactate dehydrogenase (LDH) (Table 1). The white blood cells (WBCs) and platelets (PLT) counts were within normal limits. Peripheral blood smear showed spherocytes and polychromatic red cells consistent with haemolysis. Direct and indirect Coombs' tests were positive for immunoglobulin G (IgG) specificity, while a marked prolongation of activated partial thromboplastin time (aPTT) was obvious (Table 1). Accordingly, a diagnosis of AIHA was made.

The presence of increased IgM levels in a female patient, along with the elevation of $\gamma$-GT on several occasions, prompted us to investigate for the presence of antimitochondrial autoantibodies (AMA), the hallmark for the diagnosis of primary biliary cirrhosis (PBC) [10]. Liver autoimmune serology detected AMA (titre 1/320; positive titre $>1 / 20$ ) by indirect immunofluorescence (IIF) on in-house rat multiorgan substrate panel that included kidney, liver, and stomach as we published previously using standard protocols [10]. The AMAs were also detected twice by an enhanced performance M2 IgG-isotype-specific ELISA (30 units; UNL: 20 units) according to the manufacturer's instructions (ELISA; Quanta Lite, INOVA Diagnostics, San Diego, Calif, USA) and blotting using rat liver mitochondrial fractions [10-12]. Due to the prolongation of aPTT (Table 1), investigation for lupus anticoagulant (LA), anticardiolipin antibodies (aCLAbs), and antibodies against $\beta_{2}$-glycoprotein I (anti- $\beta_{2}$-GPI) of IgM and IgG specificity was done, which revealed high positivity for APLAbs in two occasions (Table 1), at least 12 weeks apart, as dictated by the revised classification criteria for the diagnosis of antiphospholipid syndrome (APLS) $[13,14]$. Due to the above-mentioned findings as well as to the severity of AIHA, liver biopsy at this time point was considered unethical and was not performed.

In fact, both IgM and IgG aCL Abs as well as IgM anti- $\beta_{2}$ GPI Abs were positive (Table 1; ELISAs, INOVA Diagnostics, San Diego, Calif USA; cut-offs: 20 units).

A total-body computed tomography did not show abdominal or mediastinal lymphadenopathy. Serum protein electrophoresis and subsequent immunofixation revealed a monoclonal IgM light chain while bone marrow biopsy in combination with immmunohistochemical studies established the diagnosis of lymphoplasmacytic B-NHL (Figure 1).

Prednisolone ( $1 \mathrm{mg} / \mathrm{kg} /$ day) was started, and after a sixweek course, haemoglobin, reticulocytes, and LDH levels had already become normal (Table 1). In addition, till the time of this writing, the patient has received three cycles of chemotherapy every 28 days (500 mg rituximab, $2 \mathrm{mg}$ bortezomib, and $16 \mathrm{mg}$ dexamethazone). There was a marked clinical and laboratory improvement with absence of splenomegaly and decrease of IgM serum concentration

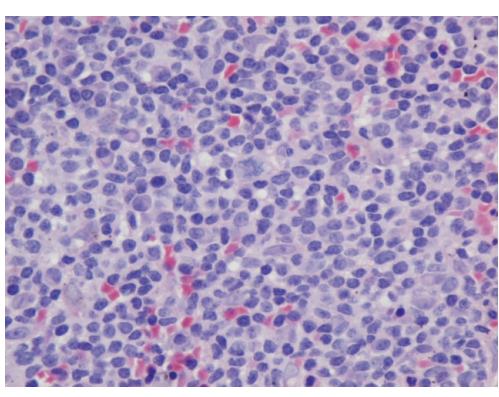

(a)

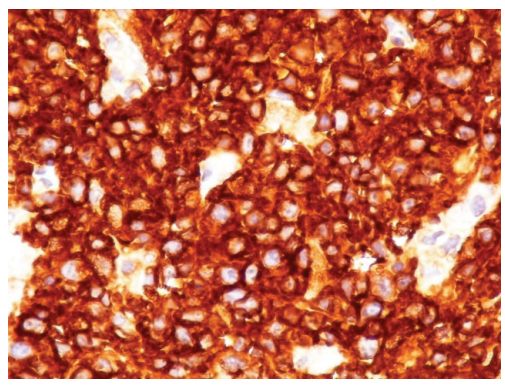

(b)

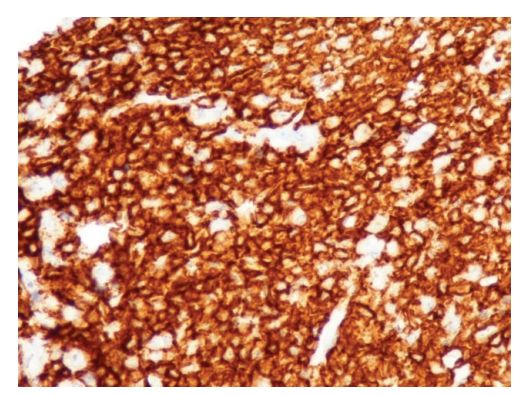

(c)

FIGURE 1: Bone marrow biopsy: Infiltration by lymphoplasmacytic B-NHL. Hematoxylin-eosin staining (a). Immunohistochemical staining for anti-CD20 (b) and anti-CD 138 (c).

(Table 1) During the whole follow-up period (10 months), no thrombotic complication or development of clinical manifestations of PBC was noted though AMA and APLAbs remained steadily positive (Table 1 ).

\section{Discussion}

To the best of our knowledge, we describe herein for the first time the simultaneous detection of AMA and APLAbs in a patient with AIHA on the ground of systemic B-NHL.

The presence of AMA with anti-M2 antibody specificity is the major hallmark for the diagnosis of $\mathrm{PBC}[10,15]$. Actually, PBC diagnosis is usually based on the presence of at least 2 out of 3 of the following criteria: elevation of cholestatic enzymes, positive titre of AMA (titre $\geq 1 / 40$ ), and characteristic liver biopsy [10, 15-17]. Clinically, the disease has a combination of characteristic symptoms including pruritus and profound fatigue though asymptomatic cases at the time of diagnosis are not infrequent as it is well known 


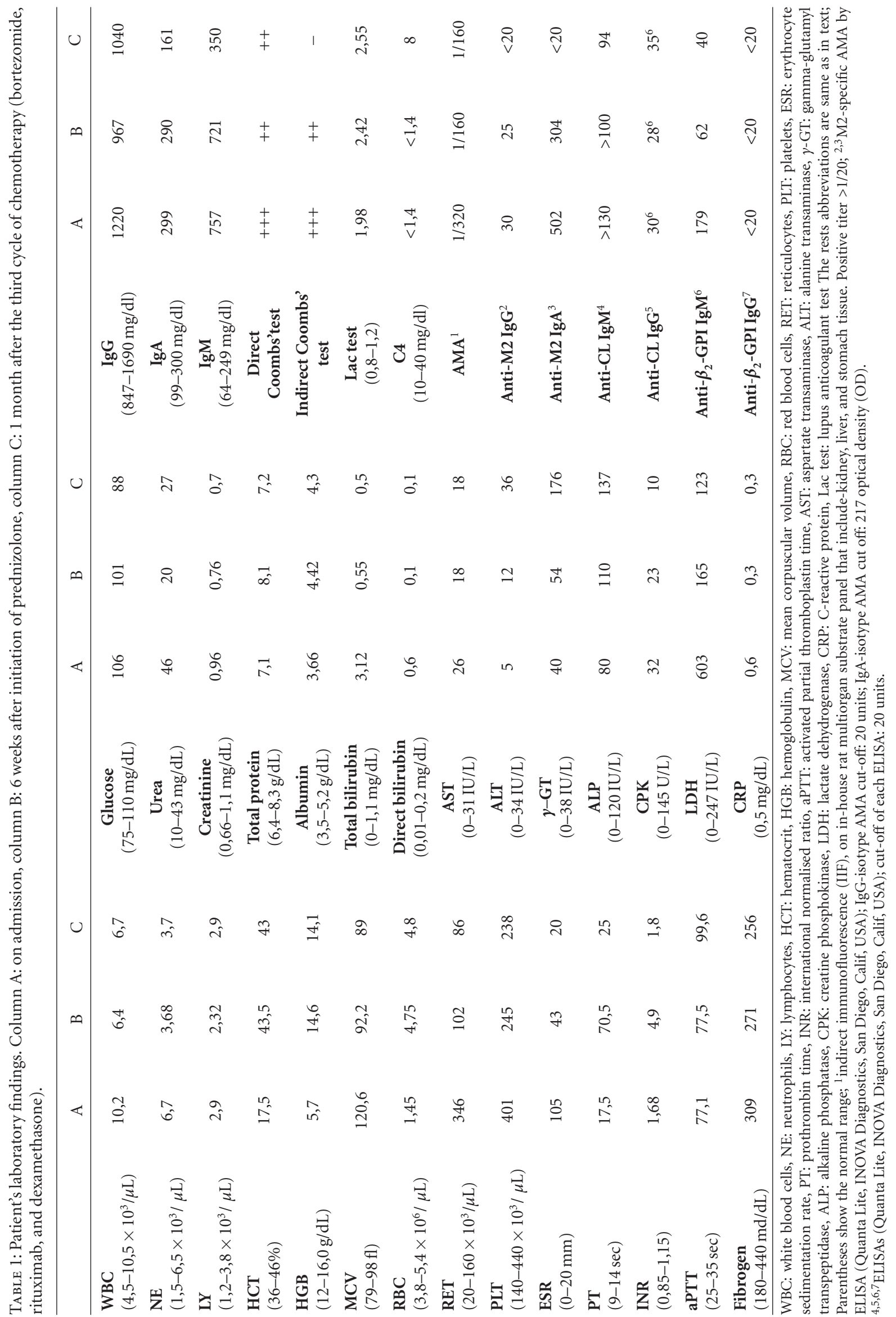


that even an isolated AMA positive test is indicative of early $\mathrm{PBC}$ and that silent disease eventually becomes clinically and biochemically evident up to 18 years after the first AMA screening [16-19]. In our case, the stable presence of AMA during followup rather excluded false positive serology during the acute setting of AIHA. However, it is not known whether PBC preceded NHL since they were diagnosed simultaneously.

Conflicting evidence exists regarding the association between PBC and NHL, while the presence of AMA without any clinical sign of liver disease, in patients with hematologic malignancies, has also been reported [6]. There are also several case reports linking PBC with NHL especially of the primary hepatic type [20-22]. However, a recent retrospective study by Panjala et al. [23] failed to confirm such an association since the estimated baseline risk of lymphoma in 2912 patients with $\mathrm{PBC}$ was as low as $<1 \%$. Of note, the latter study showed that disease involvement was predominately extrahepatic, above the diaphragm [23]. There are also case reports of splenic lymphoma with hepatic infiltration presented as AMA-positive PBC [24]. However, neither the prevalence nor the clinical significance of AMA in hematologic malignancies with liver involvement has been estimated in large series.

APLAbs have been frequently associated with NHL as well as PBC $[25,26]$. Their prevalence in patients with NHL varies from $26.6 \%$ to $41 \%$ [25, 27]. The pathogenicity of APLAbs in malignancy and in particular in NHL is not very clear: they do not seem to correlate with thrombotic events or other clinical manifestations of the APLS [25, 28], though thrombotic events associated with APLAbs can be the first manifestation of malignancy including NHL [29], while APLAbs have been suggested as independent prognostic factors in cases with aggressive NHL [27]. On the other hand, IgM and/or IgG aCLAbs have been detected in up to $40 \%$ of patients with $\mathrm{PBC}$; they were associated with more severe disease, but they rather seem to be "nonpathogenic or nonthrombogenic" (anti- $\beta_{2}$-GPI-independent) [25]. However, our patient was also IgM anti- $\beta_{2}$-GPI Abs positive which means that the IgM aCLAbs were potentially "pathogenic or thrombogenic" (co-factor dependent), and therefore, a close followup for any clinical manifestation of the APLS seems mandatory.

Finally, it is well known that AIHA is highly associated with lymphoproliferative disorders [1, 5-7]. This frequent and close association makes the exclusion of lymphoproliferative disorders essential when AIHA is diagnosed [1, 5-7]. On the contrary, one of the many extrahepatic autoimmune manifestations that PBC has been linked to is AIHA, mainly in case reports [30-32].

Overall, all the above-mentioned data indicate that, in our patient, it is not clear whether the multiple autoimmune reactions with the simultaneous detection of APLAbs, AIHA, and AMA is the cause or the effect of the underlying lymphoproliferative disorder. Goodnow [33] described the pathways and genes likely to be involved in both autoimmune diseases and lymphomas, emphasizing that both types of diseases arise as a consequence of multistep processes that eliminate the checkpoints that inhibit uncontrolled B-cell growth, including uncontrolled growth of autoimmune lymphocytes. The most prominent example is the finding that somatic and germ-line Fas mutations, which presumably interfere with apoptosis, are associated with both autoimmune diseases and lymphomas in mice and in humans, while B-cell-activating factor of the TNF family (BAFF), which enhances survival of B cells, is found to be overexpressed in Sjogren's syndrome, rheumatoid arthritis, systemic lupus erythematosus, and lymphomas [34, 35].

In conclusion, our clinical case illustrates the possibility of a common pathogenetic pathway between the loss of tolerance to self-antigens leading to autoimmune phenomena and a lymphoproliferative disorder and raises, once again, the unanswered question of which is the cause and which the effect.

\section{Acknowledgments}

The authors wish to thank Dr. Maria Ioannou, Assistant Professor of Pathology for her study of the pathological evaluation of the bone marrow biopsy of the patient.

\section{References}

[1] D. D. Alexander, P. J. Mink, H. O. Adami et al., "The non-Hodgkin lymphomas: a review of the epidemiologic literature," International Journal of Cancer, vol. 120, no. 14, supplement 12, pp. 1-39, 2007.

[2] S. A.M. van de Schans, D. J. van Spronsen, H. Hooijkaas, M. L.G. Janssen-Heijnen, and J. W.W. Coebergh, "Excess of autoimmune and chronic inflammatory disorders in patients with lymphoma compared with all cancer patients: a cancer registry-based analysis in the south of the Netherlands," Autoimmunity Reviews, vol. 10, no. 4, pp. 228-234, 2011.

[3] C. Schuetz, T. Niehues, W. Friedrich, and K. Schwarz, "Autoimmunity, autoinflammation and lymphoma in combined immunodeficiency (CID)," Autoimmunity Reviews, vol. 9, no. 7, pp. 477-482, 2010.

[4] J.-C. Souberbielle, J.-J. Body, J. M. Lappe et al., "Vitamin D and musculoskeletal health, cardiovascular disease, autoimmunity and cancer: recommendations for clinical practice," Autoimmunity Reviews, vol. 9, no. 11, pp. 709-715, 2010.

[5] R. C. Mellors, "Autoimmune disease in NZB-Bl mice. II. Autoimmunity and malignant lymphoma," Blood, vol. 27, no. 4, pp. 435-448, 1966.

[6] E. Zintzaras, M. Voulgarelis, and H. M. Moutsopoulos, "The risk of lymphoma development in autoimmune diseases: a meta-analysis," Archives of Internal Medicine, vol. 165, no. 20, pp. 2337-2344, 2005.

[7] F. Jardin, H. Lévesque, and H. Tilly, "Auto-immune manifestations in non-Hodgkin's lymphomaManifestations dysimmunitaires associées aux lymphomes," Revue de Medecine Interne, vol. 26, no. 7, pp. 557-571, 2005.

[8] L. R. Goldin and O. Landgren, "Autoimmunity and lymphomagenesis," International Journal of Cancer, vol. 124, no. 7, pp. 1497-1502, 2009.

[9] D. N. Martin, I. S. Mikhail, and O. Landgren, "Autoimmunity and hematologic malignancies: associations and mechanisms," Leukemia and Lymphoma, vol. 50, no. 4, pp. 541-550, 2009.

[10] E. I. Rigopoulou and G. N. Dalekos, "Molecular diagnostics of primary billary cirrhosis," Expert Opinion on Medical Diagnostics, vol. 2, no. 6, pp. 621-634, 2008. 
[11] S. Gabeta, G. L. Norman, C. Liaskos et al., "Diagnostic relevance and clinical significance of the new enhanced performance M2 (MIT3) ELISA for the detection of IgA and IgG antimitochondrial antibodies in primary biliary cirrhosis," Journal of Clinical Immunology, vol. 27, no. 4, pp. 378-387, 2007.

[12] E. I. Rigopoulou, D. P. Bogdanos, C. Liaskos et al., "Antimitochondrial antibody immunofluorescent titres correlate with the number and intensity of immunoblot-detected mitochondrial bands in patients with primary biliary cirrhosis," Clinica Chimica Acta, vol. 380, no. 1-2, pp. 118-121, 2007.

[13] S. Miyakis, M. D. Lockshin, T. Atsumi et al., "International consensus statement on an update of the classification criteria for definite antiphospholipid syndrome (APS)," Journal of Thrombosis and Haemostasis, vol. 4, no. 2, pp. 295-306, 2006.

[14] G. N. Dalekos, K. Zachou, and C. Liaskos, "The antiphospholipid syndrome and infection," Current Rheumatology Reports, vol. 3, no. 4, pp. 277-285, 2001.

[15] P. Invernizzi, A. Lleo, and M. Podda, "Interpreting serological tests in diagnosing autoimmune liver diseases," Seminars in Liver Disease, vol. 27, no. 2, pp. 161-172, 2007.

[16] K. D. Lindor, M. E. Gershwin, R. Poupon, M. Kaplan, N. V. Bergasa, and E. J. Heathcote, "Primary biliary cirrhosis," Hepatology, vol. 50, no. 1, pp. 291-308, 2009.

[17] R. Poupon, "Primary biliary cirrhosis: a 2010 update," Journal of Hepatology, vol. 52, no. 5, pp. 745-758, 2010.

[18] H. C. Mitchison, M. F. Bassendine, and A. Hendrick, "Positive antimitochondrial antibody but normal alkaline phosphatase: is this primary biliary cirrhosis?" Hepatology, vol. 6, no. 6, pp. 1279-1284, 1986.

[19] J. V. Metcalf, H. C. Mitchison, J. M. Palmer, D. E. Jones, M. F. Bassendine, and O. F. W. James, "Natural history of early primary biliary cirrhosis," Lancet, vol. 348, no. 9039, pp. 13991402, 1996.

[20] S. I. Sato, T. Masuda, H. Oikawa et al., "Primary hepatic lymphoma associated with primary biliary cirrhosis," American Journal of Gastroenterology, vol. 94, no. 6, pp. 1669-1673, 1999.

[21] E. Lizarralde, P. Martínez, T. Ibañez, and A. Gutiérrez, "Primary hepatic lymphoma and primary biliary cirrhosis," American Journal of Gastroenterology, vol. 95, no. 2, pp. 562$563,2000$.

[22] R. M. Prabhu, L. J. Medeiros, D. Kumar et al., "Primary hepatic low-grade B-cell lymphoma mucosa-associated lymphoid tissue (MALT) associated with primary biliary cirrhosis," Modern Pathology, vol. 11, no. 4, pp. 404-410, 1998.

[23] C. Panjala, J. A. Talwalkar, and K. D. Lindor, "Risk of lymphoma in primary biliary cirrhosis," Clinical Gastroenterology and Hepatology, vol. 5, no. 6, pp. 761-764, 2007.

[24] M. Pinelli, M. Bindi, F. Moroni, J. Rosada, and M. Castiglioni, "Antimitochondrial antibodies and non-Hodgkin lymphoma presenting as hepatobiliary disease," Leukemia and Lymphoma, vol. 47, no. 8, pp. 1699-1700, 2006.

[25] I. Genvresse, D. Lüftner, E. Späth-Schwalbe, and F. Buttgereit, "Prevalence and clinical significance of anticardiolipin and anti- $\beta 2$-glycoprotein-I antibodies in patients with nonHodgkin's lymphoma," European Journal of Haematology, vol. 68, no. 2, pp. 84-90, 2002.

[26] K. Zachou, C. Liaskos, E. Rigopoulou et al., "Presence of high avidity anticardiolipin antibodies in patients with autoimmune cholestatic liver diseases," Clinical Immunology, vol. 119, no. 2, pp. 203-212, 2006.
[27] O. Bairey, D. Blickstein, Y. Monselise et al., "Antiphospholipid antibodies may be a new prognostic parameter in aggressive non-Hodgkin's lymphoma," European Journal of Haematology, vol. 76, no. 5, pp. 384-391, 2006.

[28] C. Font, L. Vidal, G. Espinosa et al., "Solid cancer, antiphospholipid antibodies, and venous thromboembolism," Autoimmunity Reviews, vol. 10, no. 4, pp. 222-227, 2011.

[29] J. A. Gómez-Puerta, R. Cervera, G. Espinosa et al., "Antiphospholipid antibodies associated with malignancies: clinical and pathological characteristics of 120 patients," Seminars in Arthritis and Rheumatism, vol. 35, no. 5, pp. 322-332, 2006.

[30] H. Nakasone, H. Sakugawa, J. Fukuchi et al., "A patient with primary biliary cirrhosis associated with autoimmune hemolytic anemia," Journal of Gastroenterology, vol. 35, no. 3, pp. 245-249, 2000.

[31] S. J. Fuller, P. Kumar, M. Weltman, and J. S. Wiley, "Autoimmune hemolysis associated with primary biliary cirrhosis responding to ursodeoxycholic acid as sole treatment," American Journal of Hematology, vol. 72, no. 1, pp. 31-33, 2003.

[32] E. M. Yoshida, S. H. Nantel, D. A. Owen et al., "Case report: a patient with primary biliary cirrhosis and autoimmune haemolytic anaemia," Journal of Gastroenterology and Hepatology, vol. 11, no. 5, pp. 439-442, 1996.

[33] C. C. Goodnow, "Multistep pathogenesis of autoimmune disease," Cell, vol. 130, no. 1, pp. 25-35, 2007.

[34] A. Hansen, P. E. Lipsky, and T. Dörner, "B-cell lymphoproliferation in chronic inflammatory rheumatic diseases," Nature Clinical Practice Rheumatology, vol. 3, no. 10, pp. 561-569, 2007.

[35] P. Youinou, "Editorial: is BAFF the murderer in lupus?" Lupus, vol. 17, no. 7, pp. 613-614, 2008. 


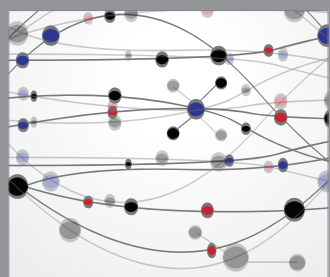

The Scientific World Journal
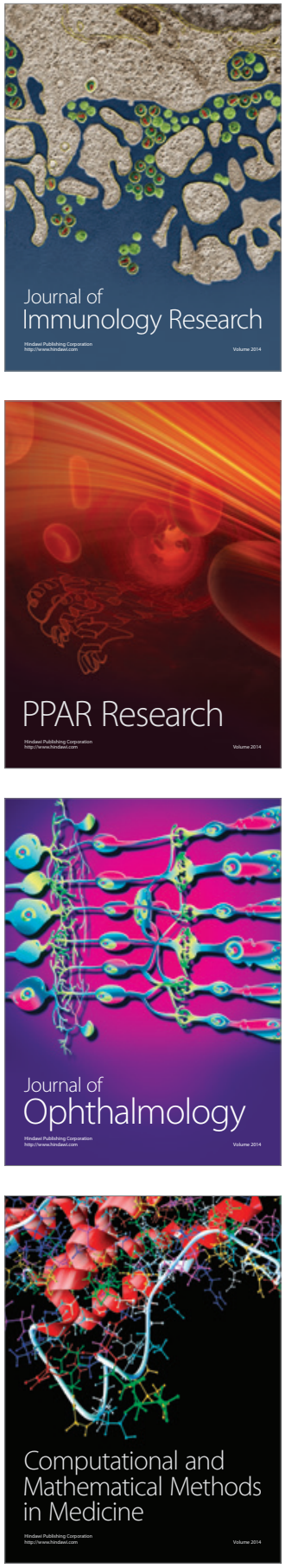

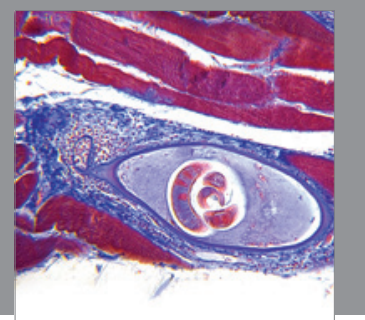

Gastroenterology

Research and Practice
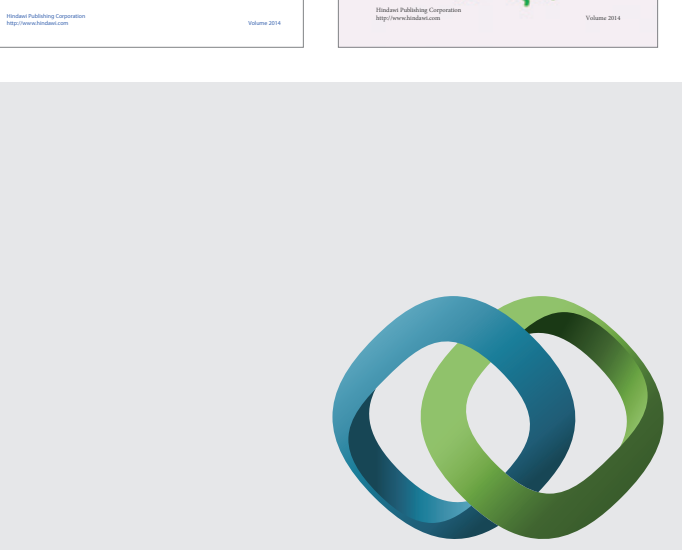

\section{Hindawi}

Submit your manuscripts at

http://www.hindawi.com
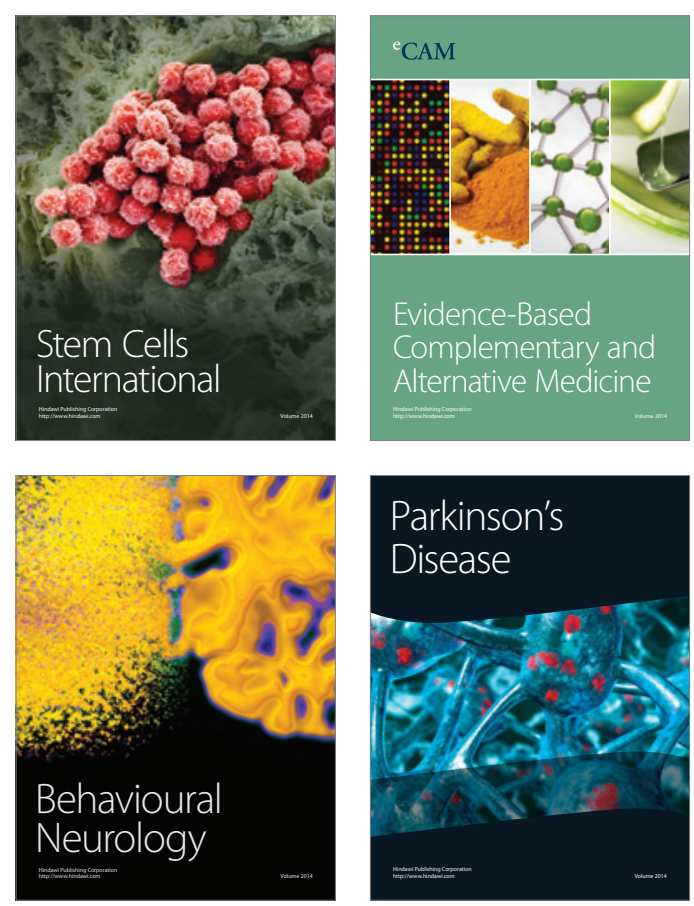

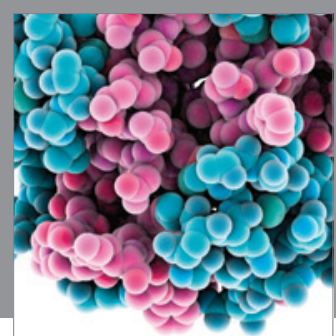

Journal of
Diabetes Research

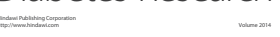

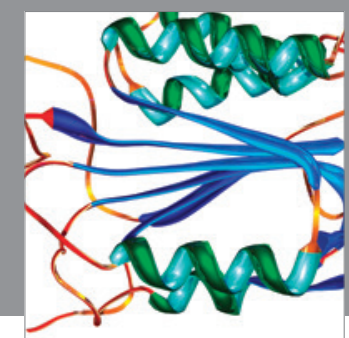

Disease Markers
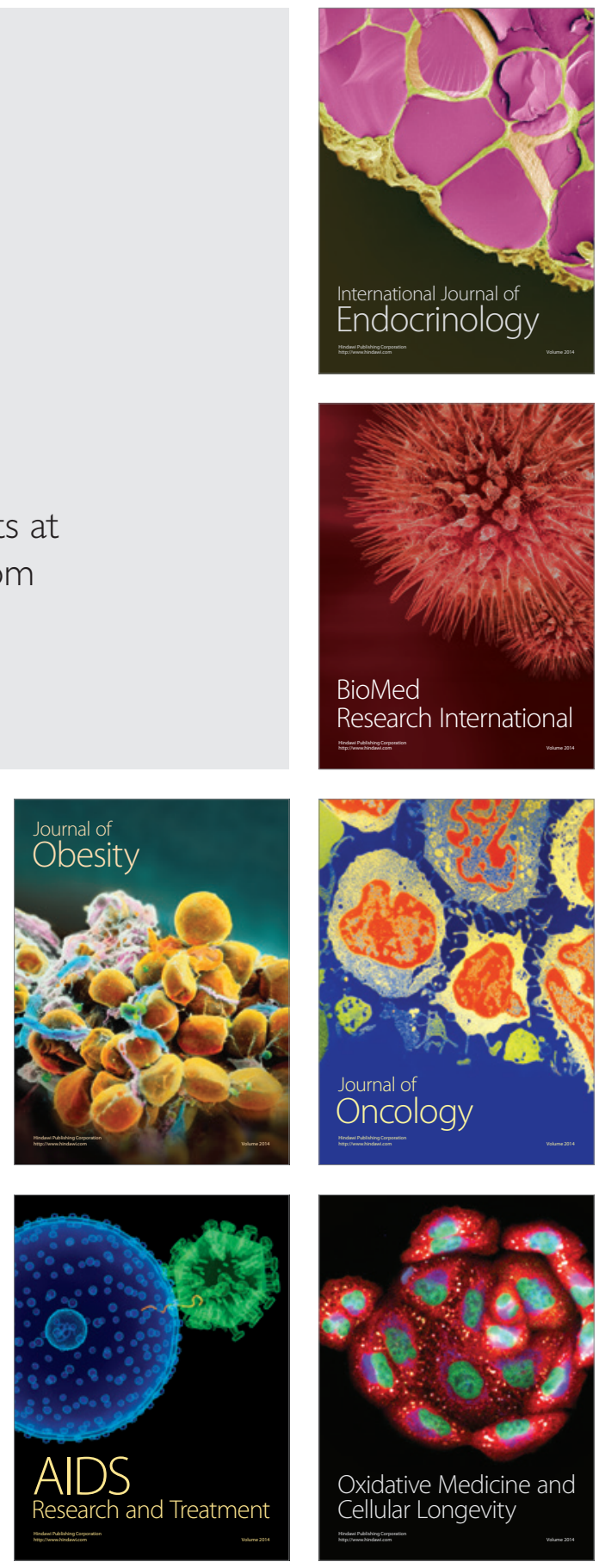